$\underline{96}$

http://dx.doi.org/10.1590/0100-2945-036/14

\title{
CARACTERIZAÇÃO QUÍMICA E FÍSICA DE FRUTOS DE CURRIOLA, GABIROBA E MURICI PROVENIENTES DO CERRADO BRASILEIRO ${ }^{1}$
}

\author{
MARESSA CALDEIRA MORZELLE ${ }^{2}$, PATRICIA BACHIEGA², ELLEN CRISTINA DE SOUZA ${ }^{3}$, \\ EDUARDO VALÉRIO DE BARROS VILAS BOAS ${ }^{4}$, MARINA LEOPOLDINA LAMOUNIER ${ }^{5}$
}

RESUMO - O bioma Cerrado apresenta uma variedade de espécies frutíferas detentoras de características sensoriais peculiares pouco exploradas científica e comercialmente. Este trabalho teve como objetivo a caracterização química, física e nutricional dos frutos de curriola (Pouteria ramiflora), gabiroba (Campomanesia cambessedeana) e murici (Byrsonima verbascifolia), nativos do Cerrado brasileiro. A gabiroba apresentou elevada quantidade de vitamina C $(383,33 \mathrm{mg} / 100 \mathrm{~g})$, bem como uma proporção significativa de polpa $(81,52 \%)$. Por sua vez, a curriola apresentou teores consideráveis de fibra bruta $(8,18 \%)$, enquanto o murici apresentou uma quantidade considerável de pectina $(746,81 \mathrm{mg} / 100 \mathrm{~g})$, lipídeos $(2,31 \%)$ e um expressivo potencial antioxidante $(56 \mathrm{mg} \mathrm{DPPH} / \mathrm{g}$ fruto). Desta forma, os resultados obtidos servem como base para pesquisas futuras no sentido da comprovação da presença de compostos bioativos e elaboração de produtos visando à agregação de valor aos frutos.

Termos para Indexação: Byrsonima verbascifolia, Campomanesia cambessedeana, Pouteria ramiflora, Antioxidantes.

\section{CHEMICAL AND PHYSICAL CHARECTERIZTION OF FRUITS FROM CERRADO: CURRIOLA, GABIROBA AND MURICI}

\begin{abstract}
The Cerrado biome features a variety of fruit species with peculiar sensory characteristics, but little explored scientifically and commercially. This study aimed the chemical, physical and nutritional characterization of curriola (Pouteria ramiflora), gabiroba (Campomanesia cambessedeana) and murici (Byrsonima verbascifolia), native fruits of the Brazilian Cerrado. Gabiroba had a high amount of Vitamin $\mathrm{C}(383.33 \mathrm{mg} / 100 \mathrm{~g})$ and showed a significant proportion of pulp $(81.52 \%)$. Curriola showed considerable crude fiber $(8.18 \%)$, while murici had a considerable amount of pectin $(746.81 \mathrm{mg} / 100 \mathrm{~g})$, lipids $(2.31 \%)$ and expressive antioxidant potential (56 mg DPPH / g fruit). These results can be used as a basis for future researches in order to improve bioactive compounds and manufacturing processes of those fruits.
\end{abstract}

Index terms: Byrsonima verbascifolia, Campomanesia cambessedeana, Pouteria ramiflora, antioxidants.

\footnotetext{
${ }^{1}$ (Trabalho 036-14). Recebido em: 23-01-2014. Aceito para publicação em: 09-10-2014.

${ }^{2}$ Universidade de São Paulo (USP), Escola Superior de Agricultura "Luiz de Queiroz", Departamento de Agroindústria, Alimentos e Nutrição. Av. Pádua Dias, 11 - Piracicaba - SP, CEP 13418-900, Tel.: 55198947 3773. E-mail: maressamorzelle@usp.br, patriciabachiega@hotmail.com

${ }^{3}$ Universidade Federal de Mato Grosso, Instituto de Ciências Exatas e da Terra (ICET). Rodovia BR-070, Km 5. Barra do Garças - MT, CEP: 78600-000, Tel.: 5566 3402-1100. E-mail: ellencsou@hotmail.com

${ }^{4}$ Universidade Federal de Lavras, Departamento de Ciência dos Alimentos. Câmpus Universitário, Caixa Postal 3037, Lavras - MG, CEP 37200-000, Tel.: 55353829 1391. E-mail: evbvboas@ufla.br

${ }^{5}$ Instituto Federal de Minas Gerais Campus Bambui. Fazenda Varginha, Caixa Postal 05, Bambuí - MG, CEP $38900-000$, Tel.: 55 37 34314936. E-mail: marina.lamounier@yahoo.com.br
} 


\section{INTRODUÇÃO}

Entre as mais ricas savanas do mundo, o bioma Cerrado constitui um imensurável patrimônio de recursos naturais renováveis, com ênfase para as espécies frutíferas exóticas detentoras de características sensoriais peculiares e intensas. Estas características únicas creditam aos frutos um potencial de exploração nacional e internacional, despertando o interesse dos consumidores e contribuindo com a busca das indústrias por inovações que proporcionem um desenvolvimento competitivo.

As plantas do Cerrado brasileiro são conhecidas como fonte de compostos de alto interesse biotecnológico, que têm aplicação tanto na indústria médica quanto na de alimentos (CARAMORI et al., 2004). As fruteiras nativas são espécies fundamentais neste ecossistema e há muitos anos são regularmente consumidas pelas populações locais tanto na forma in natura quanto como produtos processados, tais como sucos, sorvetes, pães e bolos (DAMIANI et al., 2011).

No entanto, mesmo com a crescente valorização e o emprego de produtos regionais, as informações acerca do potencial nutricional dos frutos do Cerrado são limitadas ou, muitas vezes, inexistem, sugerindo a necessidade de investimentos científicos nessa área (SOUZA et al., 2012). Essas frutas constituem uma fonte de compostos com propriedades funcionais benéficas à saúde, o que pode estimular seu uso pela indústria farmacêutica e de alimentos para o desenvolvimento de novos produtos, promovendo o desenvolvimento sustentável das regiões com as características do Cerrado (SIQUEIRA et al., 2013). Neste contexto, alguns estudos foram realizados acerca de seu efeito terapêutico (COSTA et al., 2013; GOUVEIA et al., 2013) e seu potencial na elaboração de produtos alimentícios (MORZELLE et al., 2009; MORZELLE et al., 2011; SALGADO et al., 2011; MORZELLE et al., 2012; ASSUMPÇÃO et al., 2013; SILVA et al., 2014).

Frente à escassez de informações sobre os frutos do Cerrado mencionados e seu possível potencial de inserção no mercado nacional e internacional, o presente trabalho objetivou caracterizar os frutos de curriola, murici e gabiroba in natura quanto a suas propriedades físicas, químicas, físico-químicas e valor nutricional.

\section{MATERIAL E MÉTODOS}

\section{Material Vegetal}

Foram utilizados frutos nativos de curriola (Pouteria ramiflora), murici (Byrsonima crassifolia) e gabiroba (Camponesia cambessedeana), provenientes de uma área de pastagem nativa com formação típica do Cerrado, localizada na região da cidade de Barra do Garças, Mato Grosso, Brasil. A altitude do local é de 318 metros e está localizado a $15^{\circ} 53^{\prime} 24^{\prime \prime}$ S de latitude e $52^{\circ} 15^{\prime} 24^{\prime \prime} \mathrm{W}$ de longitude.

Os frutos foram colhidos manualmente, de maneira cuidadosa, evitando danos físicos ao produto. Foi adotada rigorosa seleção quanto à maturação $\mathrm{e}$ à uniformidade do tamanho e da forma dos frutos. Para cada espécie, foram coletados aproximadamente $3 \mathrm{~kg}$ de frutos maduros. Em seguida, o material vegetal coletado foi acondicionado em caixas de isopor e imediatamente transportado para o Laboratório de Análise de Alimentos da Universidade Federal de Mato Grosso. Para as análises, foram selecionados os frutos que apresentavam ausência de injúrias e defeitos. Posteriormente, o material foi acondicionado em sacos de polietileno e conservado em freezer a $-18^{\circ} \mathrm{C}$, para posteriores análises.

\section{Determinação das características físicas}

O peso de dez frutos foi determinado em balança semianalítica digital (Mettler, modelo PC2000), e os resultados apresentados correspondem à média destes valores. Pesaram-se o fruto integral e, posteriormente, o caroço. Para o cálculo do rendimento, o resultado da diferença entre o peso da fruta e o peso do caroço foi dividido pelo peso deste último.

A cor dos frutos foi determinada em 10 diferentes pontos de 3 frutos, utilizando colorímetro (Konica Minolta Sensing, modelo CR400) com a determinação no modo CIE L*a*b*.

\section{químicas}

Determinação das características físico-

Os sólidos solúveis totais foram determinados utilizando um refratômetro de campo (Briobrix, Modelo 2 WAJ); o pH, através de um potenciômetro digital (Digimed, modelo DMPH-2), e a acidez total titulável foi determinada por meio de titulação de acordo com metodologia descrita no Instituto Adolfo Lutz (2008).

\section{Determinação da composição química}

A quantificação de açúcares redutores, não redutores e totais foi realizada pelo método de Somogyi-Nelson, de acordo com metodologia do Instituto Adolfo Lutz (2008). Os teores de pectina solúvel e total foram extraídos de acordo com a técnica de McCready e McColomb (1952) e 
determinados segundo técnicas de Blumenkrantz e Asboe-Hansen (1973). O conteúdo de vitamina C das amostras foi determinado pelo método colorimétrico (INSTITUTO ADOLFO LUTZ, 2008), e os resultados, expressos em mg de ácido ascórbico por $100 \mathrm{~g}$ de polpa. O potencial antioxidante dos frutos foi determinado com base na extinção da absorção do radical 2,2 - difenil-1-picril hidrazil (DPPH 60 $\mu \mathrm{M})$, segundo Rufino (2007).

Determinação da composição centesimal

As análises de umidade, proteínas, cinzas e extrato etéreo foram realizadas em triplicada, segundo as metodologias descritas pela AOAC (2005). A quantificação das fibras foi realizada através de um método gravimétrico, que submete as amostras a uma hidrólise ácida, de acordo com a metodologia descrita pelo Instituto Adolfo Lutz (2005). A fração glicídica ou extrato não nitrogenado calculou-se por diferença, considerando a matéria integral. Todos os resultados foram expressos em média $(n=3)$ e desvio-padrão, exceto o teor de carboidratos, que foi calculado utilizando a média obtida para os outros nutrientes.

\section{RESULTADOS E DISCUSSÃO} química

Caracterização física, química e físico-

Os resultados das características físicas, químicas e físico-químicas dos frutos estudados podem ser observados na Tabela 1 .

Os frutos de curriola (Pouteria ramiflora) são do tipo baga com casca de coloração verde, mesmo quando madura, envolvendo uma polpa branca. Geralmente apresentam formato piriforme e uma semente única envolvida por uma polpa doce, mas podem apresentar de 1 a 3 sementes (SILVA JÚNIOR, 2005). Conforme observado nos valores apresentados na Tabela 1, a casca e a semente corresponderam a $56,68 \%$ do peso total do fruto. A porção comestível, constituída pela polpa do fruto, apresentou um peso médio de 13,59 g, totalizando um rendimento de $43,32 \%$.

A gabiroba é um fruto do tipo baga com formato arredondado, de coloração que varia do verde-escuro ao amarelo e pode conter de 1 a 9 sementes (SILVA et al., 2009). Os frutos de gabiroba apresentaram o peso médio total de aproximadamente 4,00 g, das quais 1,50 g era massa comestível que inclui polpa e casca, totalizando 81,52\% de rendimento (Tabela 1 ). Os frutos analisados apresentaram um peso médio superior ao valor encontrado por Candido et al. (2009). Esta variação é comum em frutos do Cerrado, uma vez que são espécies ainda não domesticadas, havendo desta forma variação entre as matrizes para características como coloração, tamanho e peso (SILVA et al., 2012).

O murici é um fruto do tipo drupa, com mesocarpo carnoso e fino. Apresenta a casca e a polpa suculenta com uma coloração amarela intensa e sabor adocicado quando maduro (ALMEIDA et al., 1998; LORENZI, 1998). Os resultados obtidos (Tabela 1) demonstraram que o rendimento da polpa comestível do fruto, que corresponde à casca e à polpa, foi de aproximadamente $75,99 \%$. Gusmão et al. (2006) encontraram valores semelhantes $(73,63 \%)$ quanto ao rendimento de frutos de murici da região de Minas Gerais, Brasil, enquanto Araújo et al. (2009) relataram um rendimento inferior $(63,00 \%) \mathrm{em}$ frutos de murici provenientes de Alagoas. Isso pode ser justificado pela presença de maior quantidade de sementes nos frutos de acordo com as condições ambientais da região.

O rendimento é um fator-chave a ser considerado para a escolha da matéria-prima ideal pela indústria de alimentos. A gabiroba e o murici apresentaram elevado rendimento quando comparado a outras frutas como manga $(56,88 \%)$ e bacuri $(11,82 \%)$, potencializando sua utilização como matéria-prima para processamento de diversos alimentos (HILUEY et al., 2005; SILVA et al., 2010). Além disso, o elevado teor de polpa é uma das características mais desejáveis na comercialização de frutas in natura (CHITARRA; CHITARRA, 2005).

Quanto à coloração, a gabiroba apresentou uma coloração mais escura, com valor de 26,09 para L; enquanto a curriola demonstrou uma polpa de coloração clara, com valor de 67,52 para o mesmo parâmetro. Os frutos de murici caracterizam-se pela sua cor amarela, evidenciada pelo valor de 41,69 encontrado para $b^{*}$.

$\mathrm{O}$ fruto de curriola apresentou um $\mathrm{pH}$ aproximando-se do neutro $(5,93)$, enquanto os frutos de gabiroba e murici demonstraram valores de $\mathrm{pH}$ mais ácidos, de 4,25 e 4,74, respectivamente. A acidez total também foi menor no fruto de curriola quando comparado aos frutos de gabiroba e murici, estando de acordo com os resultados encontrados na medida de $\mathrm{pH}$.

De maneira geral, as três espécies analisadas apresentaram um moderado teor de SST. Valores expressivos de sólidos solúveis são valorizados pela indústria de alimentos e também para o consumo in natura, uma vez que estão intimamente relacionados a um maior rendimento durante o processamento (SANTOS et al., 2010).

Os $11,7^{\circ}$ Brix encontrados para sólidos solúveis totais em frutos de gabiroba são semelhantes 
aos $11^{\circ}$ Brix relatados por Freitas et al. (2008) para frutos de gabiroba provenientes da cidade de Goiânia-GO.

Em relação ao murici, Araújo et al. (2009) encontraram valores inferiores de sólidos solúveis $\left(9,75^{\circ}\right.$ Brix) em frutos provenientes da região de Maceió-AL. Como já descrito anteriormente, os frutos do Cerrado são espécies não domesticadas, o que pode trazer variações de seu conteúdo químico e nutricional.

Damiani et al. (2009) relataram a existência de uma relação inversa entre a quantidade de açúcares redutores e a quantidade de sacarose, o que foi observado neste trabalho, de acordo com a Tabela 1.

Dentre os frutos analisados, o murici apresentou o maior teor de pectina, sendo em sua maioria pectina insolúvel. Estes dados indicam sua potencial aplicabilidade na elaboração de geleias e sucos.

O Ministério da Saúde recomenda a ingestão diária de $60 \mathrm{mg}$ de vitamina $\mathrm{C}$ para adultos (BRASIL, 2003). A partir dos resultados obtidos (Tabela 1), pode-se inferir que a ingestão aproximada de 10 frutos de gabiroba supre a ingestão diária recomendada (IDR) para adultos. Segundo Gama et al. (2002), os frutos considerados fontes elevadas de vitamina C contêm de 100 a $300 \mathrm{mg} / 100 \mathrm{~g}$. Analisando os resultados expressos na Tabela 1, é possível afirmar que os frutos de gabiroba podem ser classificados dentro dessa categoria.

Atualmente, existe um interesse crescente quanto aos antioxidantes naturais, uma vez que evidências científicas indicam que estes compostos contribuem para a redução do risco de doenças crônicas não transmissíveis, como as cardiovasculares, câncer ou neurodegenerativas (MCCUNE et al., 2011).

A gabiroba apresentou expressivo potencial antioxidante que, possivelmente, pode estar relacionado ao seu elevado teor de vitamina $\mathrm{C}$, além de outros compostos antioxidantes como os fenólicos.

Se comparado a outros frutos, o potencial antioxidante do murici foi elevado em relação ao valor de 1,4 mg DPPH/g fruta encontrada na pitaia madura, nativa do Cerrado, madura, por Rodrigues (2010), e aos 41,14 mg DPPH/g fruto encontrados na polpa do abacaxi por Kuskoski et al. (2006), demonstrando valor expressivo de potencial antioxidante.

Siqueira et al. (2013) compararam a capacidade antioxidante, mensurada através de diferentes métodos (DPPH, FRAP e sistema betacaroteno/ácido linoleico), bem como o teor de compostos fenólicos, vitamina $\mathrm{C}$, flavonoides e antocianinas entre diferentes frutos do Cerrado (araticum, baru, cagaita, cajuzinho, guariroba, ingá, jatobá, jenipapo, jurubeba, lobeira, mangaba e tucum) e maçã-vermelha (Malus domestica). Os frutos de araticum, cagaita, cajuzinho, mangaba, lobeira, jurubeba e tucum são expressivas fontes de compostos antioxidantes em comparação à maçã. Desta forma, o consumo destes frutos poderia proteger o organismo contra o estresse oxidativo e, consequentemente, prevenir a incidência de inúmeras doenças crônicas não transmissíveis. É preciso estimular a produção, comercialização e consumo de frutas do Cerrado, o que poderia, consequentemente, incentivar o desenvolvimento sustentável da região onde esse bioma domina.

Os frutos de curriola não apresentaram uma significante atividade antioxidante $(13,69 \mathrm{mg}$ $\mathrm{DPPH} / \mathrm{g}$ fruto) quando mensurado pelo método DPPH.

O estudo da atividade antioxidante dos frutos é importante, uma vez que a relação entre alimento e saúde está sendo explorada pela comunidade científica, e os frutos do Cerrado têm recebido destaque neste cenário. Estudos complementares devem ser realizados a fim de verificar quais compostos seriam responsáveis por esta atividade nos frutos de murici e gabiroba.

\section{Composição Centesimal}

As composições centesimais dos frutos analisados podem ser verificadas na Tabela 2 .

Conforme pode ser observado na Tabela 2, os frutos de curriola apresentaram o maior teor de fibra bruta $(8,18 \%)$. A quantidade de fibra presente na curriola supera os teores encontrados em frutas convencionais, como kiwi, jabuticaba e goiabavermelha (FRANCO, 2004).

Um produto deve conter um mínimo de $3 \%$ de fibras para ser considerado uma fonte de fibras (BRASIL, 1998), ou seja, os resultados indicaram que a curriola é uma excelente fonte de fibras. Diante do exposto, a curriola, possivelmente, pode ser aplicada na elaboração de produtos integrais ou até mesmo na elaboração de barra de cereais.

A fibra bruta encontrada nos frutos de gabiroba representou $4,14 \%$ da composição total do fruto, sendo o menor teor apresentado dentre os frutos analisados. Estudos de Santos et al. (2009) relataram valores semelhantes $(3,84 \%)$ para gabiroba proveniente do Estado de Minas Gerais. A gabiroba também pode ser considerada uma fonte de fibras.

Segundo Vilas Boas (2002) e Chitarra e Chitarra (2005), quantidades semelhantes de fibras da gabiroba e do murici foram encontradas também 
em outros frutos, como coco $(4,1 \%)$, tomate $(4 \%)$, graviola $(4,31 \%)$ e goiaba-vermelha $(4,95 \%)$, enquanto valores reduzidos foram verificados em acerola $(2,49 \%)$, maçã $(2,00 \%)$, banana-nanica $(1,50 \%)$, jabuticaba $(2,06 \%)$, uva $(2,27 \%)$ e caju $(2,30 \%)$.

Quanto à concentração de resíduo mineral fixo, a gabiroba apresentou menor teor, perfazendo cerca de $0,41 \%$ da composição centesimal do fruto (Tabela 2). No entanto, o valor encontrado foi elevado quando comparado aos dados obtidos por Silva et al. (2008). Estes mesmos autores afirmaram que a reduzida quantidade de resíduo mineral fixo pode ser confirmado, em parte, pela concentração baixa de cálcio, ferro e zinco deste fruto.

O teor de cinzas encontrado nos frutos de murici (Tabela 2) foi superior aos dados encontrados no fruto por Guimarães e Silva (2008) e por Silva et al. (2008), de $0,63 \%$ e $0,78 \%$, respectivamente. A curriola apresentou o maior teor de resíduo mineral fixo dentre os frutos analisados, como pode ser observado na Tabela 2. Estudos posteriores precisam ser realizados a fim de se obter a composição mineral do fruto.

Com relação aos teores de proteínas dos frutos, a gabiroba apresentou valores inferiores aos outros frutos; o que, de acordo com Santos et al. (2007), pode ser explicado, pois os frutos da família Myrtaceae, à qual pertence este fruto, apresentam baixos teores de lipídeos e proteínas.

Os frutos de murici apresentaram teores expressivos de lipídeos. Siguemoto (2013) descreve que este fruto apresenta, em seu perfil lipídico, cerca de $65 \%$ de ácidos graxos monoinsaturados, sendo o ácido oleico o mais comum, seguido pelo ácido palmítico. O ácido graxo esteárico também pode ser detectado, porém em baixas concentrações. Além disso, os frutos de murici também possuem ácidos graxos poli-insaturados, como, por exemplo, o linoleico e o docosaexaenoico. O expressivo teor lipídico disponível no murici (Tabela 2) contribuiu para um elevado valor calórico, já que os lipídeos geram 2,3 vezes mais calorias que os carboidratos e as proteínas (SEYFFARTH; BRESSAN, 2007).

TABELA 1- Características físicas, químicas e físico-químicas de frutos de murici (Byrsonima crassifolia), curriola (Pouteria ramiflora) e gabiroba (Camponesia cambessedeana) da cidade de Barra do Garças-MT.

\begin{tabular}{cccc}
\hline Características $^{\mathbf{1}}$ & Curriola & Gabiroba & Murici \\
\hline Peso médio total (g) & $28,61 \pm 1,54$ & $1,50 \pm 0,06$ & $5,00 \pm 0,45$ \\
Massa comestível (g) & $11,83 \pm 0,85$ & $1,84 \pm 0,08$ & $6,58 \pm 0,83$ \\
Rendimento (\%) & $41,33 \pm 1,83$ & $81,52 \pm 1,35$ & $75,99 \pm 1,90$ \\
L & $67,52 \pm 0,62$ & $26,09 \pm 2,00$ & $56,38 \pm 2,79$ \\
a & $-6,08 \pm 0,62$ & $12,26 \pm 1,17$ & $4,94 \pm 1,41$ \\
b & $25,72 \pm 1,59$ & $13,23 \pm 1,51$ & $41,69 \pm 3,51$ \\
pH & $5,93 \pm 0,06$ & $4,25 \pm 0,00$ & $4,74 \pm 0,02$ \\
SST & $11,83 \pm 0,05$ & $11,70 \pm 0,00$ & $12,06 \pm 0,05$ \\
Acidez Titulável (\%) & $0,12 \pm 0,002$ & $0,19 \pm 0,004$ & $0,17 \pm 0,01$ \\
Açucares Redutores (g/100 g) & $8,86 \pm 0,49$ & $8,35 \pm 0,36$ & $2,97 \pm 0,09$ \\
Açucares não Redutores (g/100 g) & $5,22 \pm 0,55$ & $1,1 \pm 0,03$ & $0,13 \pm 0,02$ \\
Pectina Total (mg/100 g) & $382,49 \pm 40,38$ & $258,54 \pm 22,89$ & $746,81 \pm 58,05$ \\
Pectina Solúvel (mg/100 g) & $148,91 \pm 19,80$ & $131,15 \pm 9,97$ & $72,18 \pm 1,33$ \\
Vitamina C (mg/100 g) & $50,99 \pm 1,28$ & $383,33 \pm 17,62$ & $92,59 \pm 6,4$ \\
DPPH & $13,69 \pm 0,35$ & $49,00 \pm 1,38$ & $56,00 \pm 1,75$
\end{tabular}

${ }^{1}$ Para as características de peso médio total, massa comestível e rendimento, o número de repetições foi igual a 10 (n=10). Para as demais características, o número de repetições foi igual a três $(n=3)$. 
TABELA 2- Composição centesimal de gabiroba (Camponesia cambessedeana), murici (Byrsonima crassifolia) e curriola (Pouteria ramiflora) da região de Barra do Garças-MT.

\begin{tabular}{c|c|c|c|c|c|c}
\hline & $\begin{array}{c}\text { Umidade } \\
(\%)\end{array}$ & $\begin{array}{c}\text { Proteínas } \\
(\mathbf{\%}) *\end{array}$ & $\begin{array}{c}\text { Fibras } \\
(\mathbf{\%}) *\end{array}$ & $\begin{array}{c}\text { Resíduo } \\
\text { Mineral Fixo } \\
(\%)\end{array}$ & $\begin{array}{c}\text { Lipídeos } \\
(\%) *\end{array}$ & $\begin{array}{c}\text { Carboidratos } \\
(\mathbf{\%}) * *\end{array}$ \\
\hline Curriola & $70,68 \pm 1,52$ & $2,08 \pm 0,01$ & $8,18 \pm 1,08$ & $1,06 \pm 0,03$ & $0,84 \pm 0,01$ & 17,16 \\
Gabiroba & $77,02 \pm 0,40$ & $1,43 \pm 0,01$ & $4,14 \pm 0,026$ & $0,41 \pm 0,034$ & $1,32 \pm 0,02$ & 15,68 \\
Murici & $70,90 \pm 0,44$ & $1,94 \pm 0,02$ & $4,98 \pm 0,35$ & $1,02 \pm 0,05$ & $2,31 \pm 0,04$ & 18,85 \\
\hline
\end{tabular}

*Em matéria Integral /Dados apresentados como média de três repetições \pm desvio-padrão

**Em matéria Integral /Dados apresentados como média de três repetições

\section{CONCLUSÃo}

Com a caracterização dos frutos, foi possível concluir que a gabiroba apresentou uma quantidade elevada de vitamina $\mathrm{C}$ e expressiva proporção de polpa; o murici destaca-se pela capacidade antioxidante considerável quando comparado a outras frutas, e os frutos de curriola, pela quantidade de fibras. Portanto, em virtude do teor de nutrientes encontrados, o consumo desses frutos do Cerrado deve ser incentivado, já que eles fornecem uma quantidade apreciável de nutrientes e atendem às necessidades dos consumidores modernos para frutas ricas em compostos antioxidantes e fibras.

\section{REFERÊNCIAS}

ALMEIDA, S.P.; PROENÇA, C.E.B.; SANO, S.M.; RIBEIRO, J.F. Cerrado: espécies vegetais úteis. Planaltina: EMBRAPA, 1998. 464p.

AOAC - Association of Official Analytical Chemists. Official methods of analysis, $16^{\text {th }}$ ed. Arlington, 2005. 1115p.

ARAÚJO, R.R.; SANTOS, E.D.; LEMOS, E.E.P.; ALVES, R.E. Caracterização biométrica de frutos e sementes de genótipos de murici (Byrsonima verbascifolia (L.) Rich.) do tabuleiro costeiro de Alagoas. Revista Caatinga, Mossoró, v.22, n.3, p.224-228, 2009.

ASSUMPÇÃO, C.F.; BACHIEGA, P.; SANTANA, A.T.M.C.; MORZELLE, M.C.; VILAS BOAS, B.M.; SOUZA, E.C. Néctar misto de mangaba (Hancoria speciosa Gomes) e cagaita (Eugenia dysenterica): perfil sensorial e características físico-químicas. Revista Brasileira de Produtos Agroindustriais, Campina Grande, v.15, n.3, p.219-224, 2013.
BLUMENKRANTZ, N.; ASBOE-HANSEN, G. New method for quantitative determination of uronic acids. Analytical Biochemistry, Barking, v.54, n.2, p.484-489, 1973.

BRASIL. Agência Nacional de Vigilância Sanitária. Resolução ANVISA/MS RDC n 360, de 23 de dezembro de 2003. Regulamento Técnico sobre Rotulagem Nutricional de Alimentos Embalados. Diário Oficial da União, Brasília-DF, 26 dez. 2003. Seção 1.

BRASIL. Portaria n॰ 27, de 13 de janeiro de 1998. Regulamento técnico sobre a informação nutricional complementar. Anvisa. Disponível em: <http://www.anvisa.gov.br $>$. Acesso em: 10 abr. 2012.

CANDIDO, T.L.N.; FREITAS, J.B.; SILVA, M.R. Efeito da adição de xarope de glicose nas propriedades físico-químicas e aceitabilidade do doce de gabiroba. Nutrire, São Paulo, v.34, n.2, p.1-10, 2009.

CARAMORI, S.S.; LIMA, C.S.; FERNANDES, K.F. Biochemical characterization of selected plant species from Brazilian savannas. Brazilian archives of biology and technology and International Journal, Curitiba, v.47, n.2, p.253-259, 2004.

CHITARRA, M.I.F.; CHITARRA, A.B. Pós-colheita de frutos e hortaliças: fisiologia e manuseio. 2. ed. Lavras: FAEPE, 2005. 785p.

COSTA, A.V.; CALÁBRIA, L.K.; FURTADO, F.B.; GOUVEIA, N.M.; OLIVEIRA, R.J. da S.; OLIVEIRA, V.N.; BELETTI, M.E.; ESPINDOLA, F.S. Neuroprotective effects of Pouteria ramiflora (Mart.) Radlk (Sapotaceae) extract on the brains of rats with streptozotocin-induced diabetes. Metabolic Brain Disease, Montreal, v.28, n.3, p.411-419, 2013. 
DAMIANI, C.; VILAS BOAS, E. de V. de B.; ASQUIERI, E.R.; LAGE, M.E.; OLIVEIRA, R.A.; SILVA, F.A.; PINTO, D.M.; RODRIGUES, L.J.; SILVA, E.P.; PAULA, N.R.F. Characterization of fruits from the savanna: araça (Psidium guinnensis Sw.) and marolo (Annona crassiflora Mart.). Ciência e Tecnologia de Alimentos, Campinas, v.31, n.3, p.723-729, 2011.

DAMIANI, C.; VILAS BOAS, E.V.B.; SOARES JÚNIOR, M.S.; CALIARI, C.; PAULA, M.L.; ASQUIERI, E.R. Avaliação química de geleias de manga formuladas com diferentes níveis de cascas em substituição à polpa. Ciência e Agrotecnologia, Lavras, v.33, n.1, p.177-184, 2009.

FRANCO, G. Tabela de composição química de alimentos. 9. ed. São Paulo: Atheneu, 2004. 307p.

FREITAS, J.B.; CANDIDO, T.L.N.; SILVA, M.R. Geleia de gabiroba: avaliação da aceitabilidade e características físicas e químicas. Pesquisa Agropecuária Tropical, Goiânia, v.38, n.2, p.8794, 2008.

GAMA, R.S.A.; TEIXEIRA, M.C.D.; ALMEIDA, E.N.; NÓBREGA, J. Determinação e distribuição de ácido ascórbico em três frutos tropicais. Eclética Química, Araraquara, v.27, n.1, p.0, 2002.

GOUVEIA, N.M.; ALBUQUERQUE, C.L.; ESPINDOLA, L.S.; ESPINDOLA, F.S. Pouteria ramiflora extract inhibits salivary amylolytic activity and decreases glycemic level in mice. Anais da Academia Brasileira de Ciências, Rio de Janeiro, v.85, n.3, p.1141-1148, 2013.

GUIMARÃES, M.M.; SILVA, M.S. Valor nutricional e características químicas e físicas de frutos de murici-passa (Byrsonima verbascifolia). Ciência e Tecnologia de Alimentos, Campinas, v.28, n.4, p.817-821, 2008.

GUSMÃO, E; VIEIRA, F. de A.; FONSECA, E.M. da. Biometria de frutos e endocarpos de murici (Byrsonima verbascifolia Rich. Ex A. Juss). Cerne, Lavras, v.12, n.1, p.84-91, 2006.

HILUEY, L.J.; GOMES, J.P.; ALMEIDA, F. de A.C.; SILVA, M.S.; ALEXANDRE, H.V. Avaliação do rendimento do fruto, cor da casca e polpa de manga tipo espada sob atmosfera modificada. Revista Brasileira de Produtos Agroindustriais, Campina Grande, v.7, n.2, p.151-157, 2005.
INSTITUTO ADOLFO LUTZ. Métodos físicoquímicos para análise de alimentos. 4. ed. São Paulo: Instituto Adolfo Lutz, 2008. 1.020p.

INSTITUTO ADOLFO LUTZ. Normas analíticas do Instituto Adolfo Lutz: métodos químicos e físicos para análise de alimentos. 4. ed. Brasília: Instituto Adolfo Lutz, 2005. 1018 p.

KUSKOSKI, E.M.; ASUERO, A.G.; MORALES, M.T.; FETT, R. Frutos tropicais silvestres e polpas de frutas congeladas: atividade antioxidante, polifenóis e antocianinas. Ciência Rural, Santa Maria, v.36, n.4, p.1293-1287, 2006.

LORENZI, H. Árvores brasileiras: Manual de identificação e cultivo de plantas arbóreas nativas do Brasil. 2. ed. Nova Odessa: Plantarium, 1998. 480p.

MCCREADY, P.M.; MCCOMB, E.A. Extraction and determination of total pectic material in fruits. Analytical Chemistry, Washington, v.24, n.12, p.1986-1988, 1952.

MCCUNE, L.M.; KUBOTA, C.; STENDELLHOLliS, N.R.; THOMSON, C.A. Cherries and health: a review. Critical Reviews in Food Science and Nutrition, Cleveland, v.51, n.1, p.1-12, 2011.

MORZELLE, M.C.; LAMOUNIER, M.L.; SOUZA, E.C.; SALGADO, J.M.; VILAS BOAS, E.V. de B. Caracterização físico-química e sensorial de sorvetes à base de frutos do cerrado. Revista do Instituto de Laticínios "Cândido Tostes", Juiz de Fora, v.67, n.387, p.70-78, 2012.

MORZELLE, M.C.; SOUZA, E.C.; ASSUMPÇÃO, C.F.; FLORES, J.C.J.; OLIVEIRA, K. A. de M. Agregação de valor a frutos de ata através do desenvolvimento de néctar misto de maracujá (Passiflora edulis Sims) e ata (Annona squamosa L.). Alimentos e Nutrição, Araraquara, v.20, n.3, p.389-393, 2009.

MORZELLE, M.C.; SOUZA, E.C.; ASSUMPÇÃO, C.F.; VILAS BOAS, B.M. Desenvolvimento e avaliação sensorial de néctar misto de maracujá (Passiflora edulis Sims) e araticum (Annona crassiflora). Revista Brasileira de Produtos Agroindustriais, Campina Grande, v.13, n.2, p.131135, 2011. 
R O D R I GUES, L.J. Desenvolvimento e processamento mínimo de pitaia nativa (Selenicereus setaceus Rizz) do cerrado brasileiro. 2010. 164 f. Tese (Doutorado em Ciência dos Alimentos) - Universidade Federal de Lavras, Lavras, 2010.

RUFINO, M.S.M. Determinação da atividade antioxidante total em frutas pela captura do radical livre DPPH. Fortaleza: Embrapa Agroindústria Tropical, 2007. p.4 (Comunicado Técnico, 127).

SALGADO, J.M.; RODRIGUES, B.S.; DONADOPESTANA, C.M.; DIAS, C.T. dos S.; MORZELLE, M.C. Cupuassu (Theobroma grandiflorum) peel as potential source of dietary fiber and phytochemicals in whole-bread preparations. Plant Foods for Human Nutrition, New York, v.66, n.1, p.384-390, 2011.

SANTOS, M.B.; CARDOSO, R.L.; FONSECA, A.A.O.; CONCEIÇÃO, M.N. Caracterização e qualidade de frutos de umbu-cajá (Spondias tuberosa X S. mombin) provenientes do recôncavo sul da Bahia. Revista Brasileira de Fruticultura, Jaboticabal, v.32, n.4, p.1089-1097, 2010.

SANTOS, M.S.; CARNEIRO, P.I.B.; WOSIACKI, G.; PETKOWICZ, C.L.O.; CARNEIRO, E.B.B. Caracterização físico-química, extração e análise de pectinas de frutos de Campomanesia xanthocarpa B. (Gabiroba). Semina: Ciências Agrárias, Londrina, v.30, n.1, p.101-106, 2009.

SANTOS, M.S.; PETKOWICZ, C.L.O.; WOSIACKI, G.; NOGUEIRA, A.; CARNEIRO, E.B.B. Caracterização do suco de araçá-vermelho (Psidium cattleianum Sabine) extraído mecanicamente e tratado enzimaticamente. Acta Scientiarum, Maringá, v.29, n.5, p.617-621, 2007.

SEYFFARTH, A.S.; BRESSAN, J. Os alimentos: calorias, macronutrientes e micronutrientes. In: GOVEIA, G.R. Manual do Profissional. São Paulo: Sociedade Brasileira de Diabetes, 2007. cap.1, 7p.

SIGUEMOTO, E.S. Composição nutricional e propriedades funcionais do murici (Byrsonima crassifólia) e da moringa (Moringa oleífera). 2013. 124 f. Dissertação de Mestrado (Mestrado em Nutrição e Saúde Pública) - Faculdade de Saúde Pública, Universidade de São Paulo, São Paulo, 2013.
SILVA JÚNIOR, M.C. 100 árvores do cerrado: guía de campo. Brasília: Editora Rede de Sementes do Cerrado, 2005. 278p.

SILVA, E.P.; SIQUEIRA, H.H.; LAGO, R.C.; ROSELL, C.M.; VILAS BOAS, E.V. de B. Developing fruit-based nutritious snack bars. Journal of The Science of Food and Agricultural, London, v.94, n.1, p.52-56, 2014.

SILVA, E.P.; VILAS BOAS, E.V. deB.; RODRIGUES, L.J.; SIQUEIRA, H.H. Caracterização física, química e fisiológica da gabiroba (Campomanesia pubescens) durante o desenvolvimento. Ciência e Tecnologia de Alimentos, Campinas, v.29, n.4, p.803-809, 2009.

SILVA, F.H.L.; FERNANDES, J.S.C.; ESTEVES, E.A.; TITON, M.; SANTANA, R.C. Populações, matrizes e idade da planta na expressão de variáveis físicas em frutos do pequizeiro. Revista Brasileira de Fruticultura, Jaboticabal, v.34, n.3, p.806-813, 2012.

SILVA, M.R.; LACERDA, D.B.C.L.; SANTOS, G.G.; MARTINS, D.M.O. Composição química de frutos nativos do cerrado. Ciência Rural, Santa Maria, v.38, n.6, p.1790-1793, 2008.

SIQUEIRA, E.M. de A.; ROSA, F.R.; FUSTINONI, A.M.; SANT'ANA, L.P.; ARRUDA, S.F. Brazilian savanna fruits contain higher bioactive compounds content and higher antioxidant activity relative to the conventional red delicious apple. Plos One, Cambridge, v.8, n.8, p.1-7, 2013.

SOUZA, P.M.; ELIAS, S.T.; SIMEONI, L.A.; PAULA, J.E.; GOMES, S.M.; GUERRA, E.N.S.; FONSECA, Y.M.; SILVA, E.C.; SILVEIRA, D.; MAGALHÃES, P.O. Plants from Brazilian cerrado with potent tyrosinase inhibitory activity. Plos One, Cambridge, v.7, n.11, p.1-7, 2012.

VILAS BOAS, E.V. de B. Qualidade de alimentos vegetais. Lavras: Fundação de Apoio, Pesquisa e Extensão ao Ensino da UFLA, 2002. 65p. 